\title{
Analysis of near-separatrix motion in planetary systems
}

\author{
Su Wang and Ji-Lin Zhou \\ Department of Astronomy, Nanjing University, \\ 210093, Nanjing, China \\ email: suwang@nju.edu.cn,zhoujl@nju.edu.cn
}

\begin{abstract}
Near-separatrix motion is a kind of motion of two planets with their relative apsidal longitude near the boundary between libration and circulation. Observed multiple planetary systems seem to favor near-separatrix motions between neighboring planets. In this report, we study the probability that near-separatrix motion occurs with both the linear secular system and full three-body systems. We find that generally the ratio of near-separatrix motion is small unless the eccentricities of the two planets differ from each other by an order of magintude, or they are in mean motion resonance. To explore the dynamical procedures causing the nearseparatrix motion, we suppose a modification to scattering model by adding a mass-accretion process during the protoplanet growth. Statistics on the modified scattering model indicate that the probability of the final planet pairs in near-separatrix motion is high $(\sim 85 \%)$, which may explain the high occurrence of near-separatrix motions in observed planetary systems.
\end{abstract}

Keywords. methods: analytical, methods: n-body simulations, (stars:) planetary systems

\section{Introduction}

To date, around 260 extra-solar planets are detected, among them there are 25 multiple planet systems. Statistical properties show that although planets with $a<0.05$ AU have almost circular orbits, eccentricities in the range 0-0.8 are common(Marcy etal. 2005, Papaloizou \& Terquem 2006). Various mechanisms have been proposed to generate large eccentricities after planet formation. e.g., planet-planet scattering (e.g. Ford et al., 2005, Zhou et al. 2007), resonance trapping of planets during migration (e.g., Kley et al. 2004), etc..

The apsidal alignment is a configuration believed to favor the stability of a planet system, especially for planets on highly eccentric orbits(Zhou \& Sun 2003). Based on the new catalog of exoplanets(Butler et al. 2006), Barnes \& Greenberg (2006) studied the observed multiple-planet systems and found that the relative apsidal longitude $(\Delta \varpi)$ of a large fraction of systems lie near the boundary between libration and circulation motions (where the authors called near-separatrix motion), and the systems that exhibit libration were rare. A plausible scenario generating near-separatrix motions is the planetscattering model. In this model the eccentricities of the two planets are excited by a third planet's close encounters and ejection, resulting in a two-planet system in near-separatrix motion, a configuration similar to that of the upsilon Andromedae system( Ford et al. 2005). However, the statistics of the occurrence rate of near-separatrix motions in this model is too low $(\sim 10 \%)$ as compared to that derived from observed systems $(\sim 40 \%$, Barnes 2008).

To solve the paradox and to seek the origin of near-separatrix motion, in this report we investigate the probability that the near-separatrix motion occurs in a two-planet system,

http://exoplanet.eu/ 
with both analytical approach and numerical simulations. The planet-planet scattering model leading to this motion is also revised. In $\S 2$ we analyse the two-planet system with the secular perturbation theory. Results from N-body simulations are present in $\S 3$. In $\S 4$ we modify the classical scattering model by adding a mass-accretion process, i.e., the additional planet grows from 10 Earth mass to Jupiter mass by accreting nearby gas. Statistics for the outcomes of this refined scattering model is studied, with special attentions paid on the cause of near-separatrix motion. We present our conclusions and discussions in $\S 5$.

\section{Analysis from secular perturbation theory}

We set up a model planetary system with a solar-mass star $\left(m_{0}=1 M_{\odot}\right)$ and two Jupiter-mass planets $\left(m_{1}=m_{2}=1 M_{J}\right)$. Suppose $a_{i}, e_{i}, \varpi_{i}, M_{i}(i=1,2)$ are the semimajor axis, eccentricity, longitude of pericenter and mean anomaly of the orbit of $m_{i}$, respectively. We fix the initial semi-major axis of the inner planet $\left(a_{10}=1 \mathrm{AU}\right)$ as the unit length, and vary the initial position of the outer planet between 1.3AU and 3AU. The inner boundary 1.3AU is chosen to avoid Hill unstable region of $m_{1}$ (Gladman 1993).

Based on the definition of near-separatrix motion (Barnes \& Greenberg, 2006a,2006b) we choose the minimum value of $\left(e_{1} e_{2}\right)$ as a criterion of whether a planet-pair is in nearseparatrix motion or not. According to the theory of linear secular perturbation(e.g., Zhou \& Sun 2003), the criterion during the evolution can be written as

$$
\left(e_{1} e_{2}\right)_{\text {min }}=\left|\frac{\left(\rho_{1}+\rho_{2}\right) F G-\left|\rho_{1} F^{2}+\rho_{2} G^{2}\right|}{\left(\rho_{1}-\rho_{2}\right)^{2}}\right|,
$$

where

$$
\begin{aligned}
& F=\left(\rho_{2}^{2} e_{10}^{2}-2 \rho_{2} e_{10} e_{20} \cos \Delta \varpi_{0}+e_{20}^{2}\right)^{1 / 2}, \\
& G=\left(\rho_{1}^{2} e_{10}^{2}-2 \rho_{1} e_{10} e_{20} \cos \Delta \varpi_{0}+e_{20}^{2}\right)^{1 / 2}, \\
& \rho_{1}=\frac{1}{2 c_{0}}\left[1-\xi-\sqrt{(1-\xi)^{2}+4 c_{0} \xi}\right], \\
& \rho_{2}=\frac{1}{2 c_{0}}\left[1-\xi+\sqrt{(1-\xi)^{2}+4 c_{0} \xi}\right],
\end{aligned}
$$

and $\xi \approx \frac{m_{1}}{m_{2}} \alpha^{1 / 2}, c_{0}=b_{3 / 2}^{(2)}(\alpha) / b_{3 / 2}^{(1)}(\alpha), \alpha=a_{1} / a_{2}<1$, where $b_{3 / 2}^{(i)}(\alpha)(i=1,2)$ are the Laplace coefficients.

To show the situation in the whole plane of $\left(e_{10}, e_{20}\right)$, we express the criterion in the forms of $\eta=\left(e_{1} e_{2}\right)_{\min } / e_{10}^{2}$ or $\eta^{\prime}=\left(e_{1} e_{2}\right)_{\min } / e_{20}^{2}$. Figure 1a shows the result from equation (2.1), where the boundary lines, defined in the following equations, divide the whole region into two parts (Zhou \& Sun 2003 based on Laughlin et al. 2002),

$$
\frac{e_{20}}{e_{10}}=\frac{2 \rho_{1} \rho_{2}}{\rho_{1}+\rho_{2}} \cos \Delta \varpi_{0}, \quad \text { or } \quad \frac{e_{20}}{e_{10}}=\frac{\rho_{1}+\rho_{2}}{2} \frac{1}{\cos \triangle \varpi_{0}} .
$$

The plot of $\eta^{\prime}$ in the case of $e_{10}<e_{20}$ is similar. Since in secular dynamics, $e_{1}>e_{2}$ or $e_{1}<e_{2}$ alternates, we can always choose suitable epoch to let $e_{10}>e_{20}$, thus we neglect the discussion of $\eta^{\prime}$.

We find that the boundary line defined above, equivalent to $\eta=0$, is in the middle of the $\eta<0.1$ region. Therefore, around the boundaries, the near-separatrix motion region may be defined by the value of $\eta$, for example $\eta<0.1$ or $\eta<0.01$. Figure 2 shows the variation of ratio of near-separatrix region in the whole plane of $\left(\Delta \varpi_{0}, e_{20} / e_{10}\right)$ in Fig.1a. As we can see, the probability that a planet system fall in the near-separatrix motion 

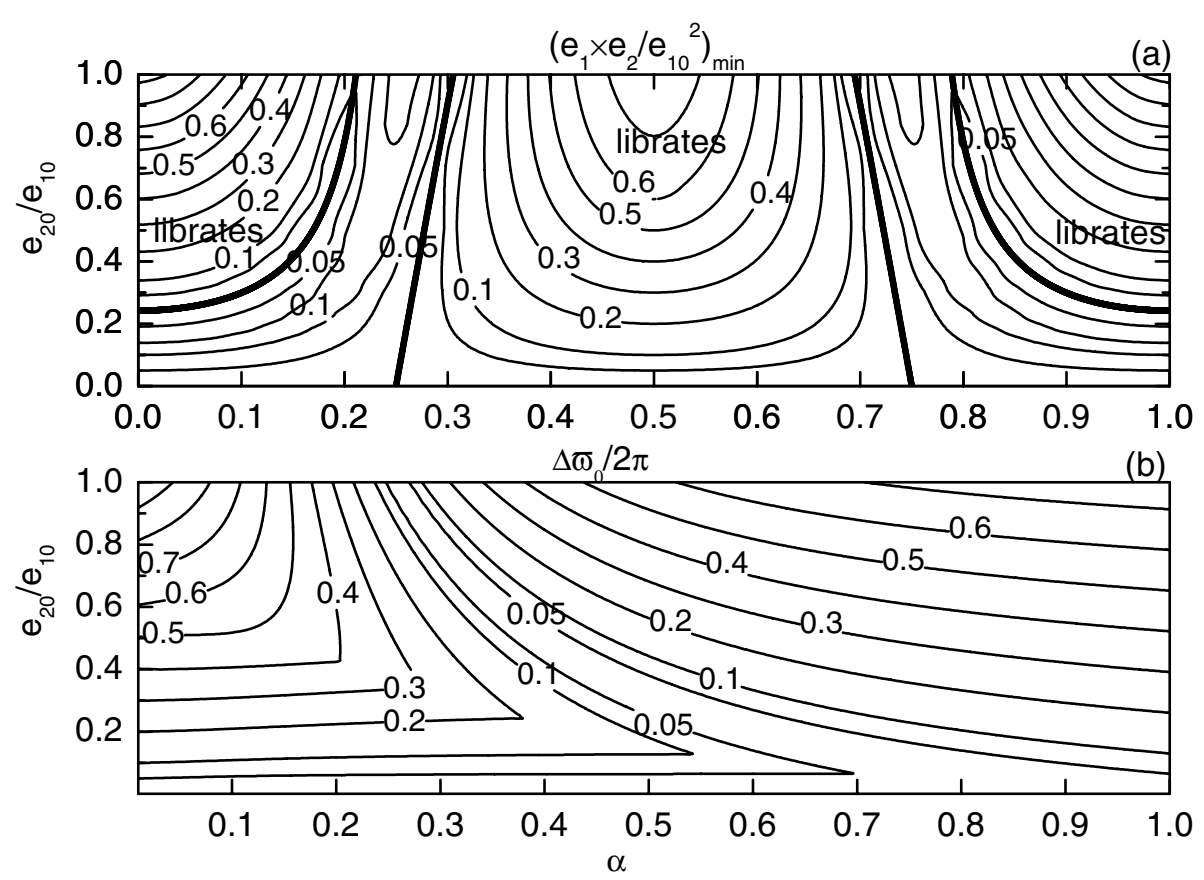

Figure 1. (a) Contours of $\eta$ changed with $e_{20} / e_{10}$ and $\Delta \varpi_{0}(\alpha=0.5)$, the wide lines represent the criterion lines for libration and circulation. (b) Contours of $\eta$ changed with $e_{20} / e_{10}$ and $\alpha$ $\left(\Delta \varpi_{0}=2 \pi / 9\right)$.

is very small in secular motion. But as long as $e_{20} / e_{10} \leqslant 0.1, \eta$ is smaller than 0.1 in most cases. With the increase of $e_{20} / e_{10}$, the area of near-separatrix region decreases. It means that, as the initial ratio of the eccentricity becomes smaller, the opportunities for the system suffering near-separatrix motion becomes larger. Thus, to obtain small values of $e_{20} / e_{10}$ is a key factor for the two-planet system to fall in near-separatrix motion.

\section{3-body simulations}

In this section, we study the evolution of two planets in a full three-body model to reveal the occurrence of near-separatrix motion. Considering a model planetary system with a solar-mass star and two Jupiter-mass planets, we carry out a series of simulations with different $e_{20} / e_{10}, \alpha$ and $\triangle \varpi_{0}$. Initially all the orbits are coplanar and the semimajor axis of the inner planet is fixed at $1 \mathrm{AU}$. The results are summarized in Figure 3. From Figure 3 we can find that the result is quite similar to that from the secular perturbation theory except near the positions of mean motion resonances between the two planets, where the secular dynamics approximation fails. In these resonant locations, the value of $\eta$ is smaller than that from the linear secular dynamics. In the plot the most obvious location is the $3: 1$ resonance at $a_{20}=2.1 \mathrm{AU}(\alpha=0.48)$. Therefore, resonance configuration seems to reduce the values of $\eta$. In order to study this phenomenon in more details, we compare two systems. The first one is in mean motion resonance initially, with $a_{20}=2.1 \mathrm{AU}$, while the second one is in non-resonance region, with $a_{20}=2.3 \mathrm{AU}$. Other 

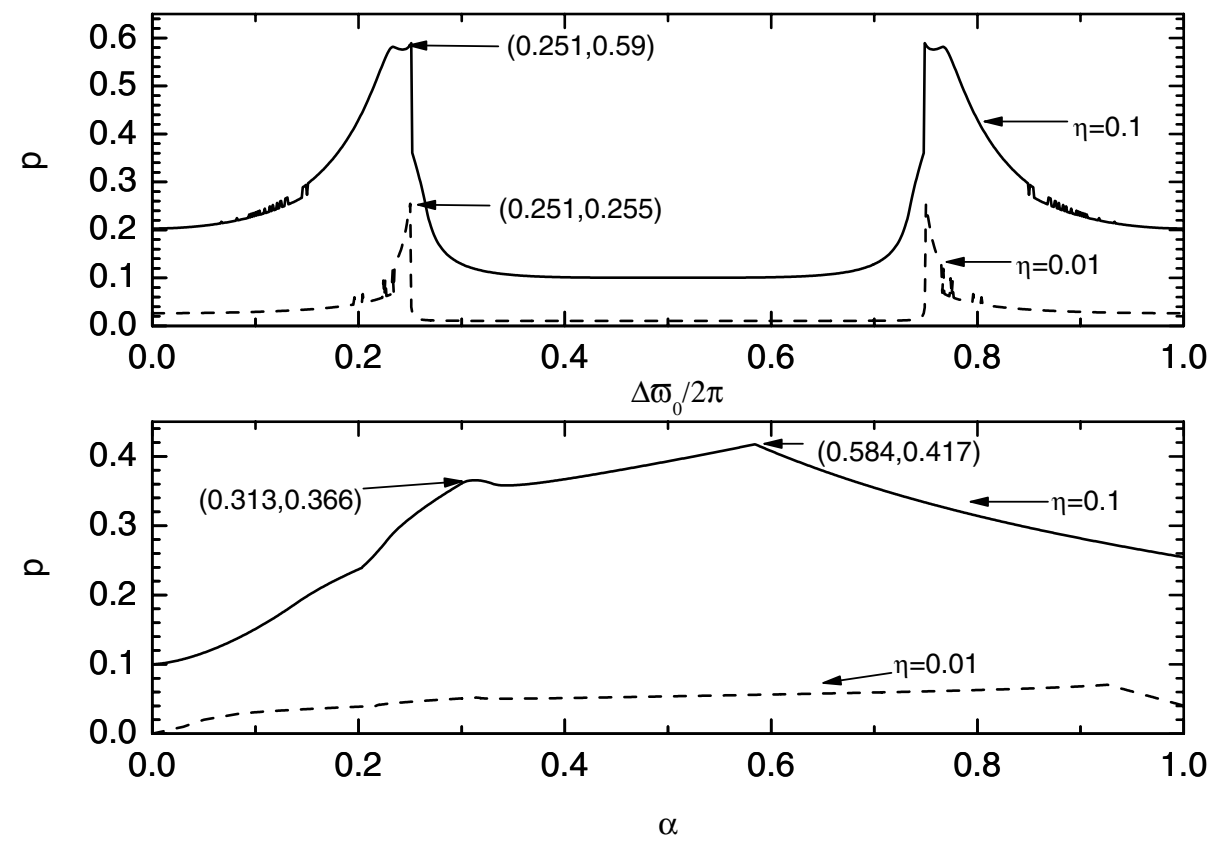

Figure 2. Ratio of near-separatrix region in the whole plane of $\left(\Delta \varpi_{0}, e_{20} / e_{10}\right)$ in Fig.1a changed with $\alpha$ and $\Delta \varpi_{0}$.

initial conditions are the same, i.e., $a_{10}=1 \mathrm{AU}, e_{10}=0.2, e_{20}=0.1$. Figure 4 a shows the evolution of the eccentricities in the system of mean motion resonance while Figure $4 \mathrm{~b}$ presents eccentricity evolution of non-resonance system. The visible difference between the two systems is that one of the eccentricities of the resonance system always stays in lower values than the one in non-resonance. As a result, the value of $\left(e_{1} e_{2}\right)_{\text {min }}$ is $8.67 \times 10^{-4}$ in resonance system. In comparison, $\left(e_{1} e_{2}\right)_{\min }=0.013$ in the non-resonance system. We checked lots of orbits and confirm this scenario. Thus systems initially in a mean motion resonance seems to be easy to fall in near-separatrix motion.

\section{Scattering process}

Ford et al. (2005) proposed a planet-planet scattering model that could generate nearseparatrix motion. In this model, a third planet is added to the previous two-planet system. Due to the gravitational perturbation, the added planet that is initially put on a circular orbit was scattered out, leaving two planets in a near-separatrix motion. This scenario requires that the added planet initially on a circular orbit must be unstable.

To achieve such an initial state, we suppose a reasonable situation as follows. During the formation of gas giants, a protoplanetary embryo will form through the cohesive collisions between planetesimals. When the protoplanet grows to a critical mass $\left(\sim 10 M_{\oplus}\right)$, a long period of stage named quasi-hydrostatic accretion sets in. As the gas envelop mass becomes comparable to the core mass, a runaway gas accretion occurs until the gas giant reaches the present mass (Pollack et al. 1996). The final stage of 

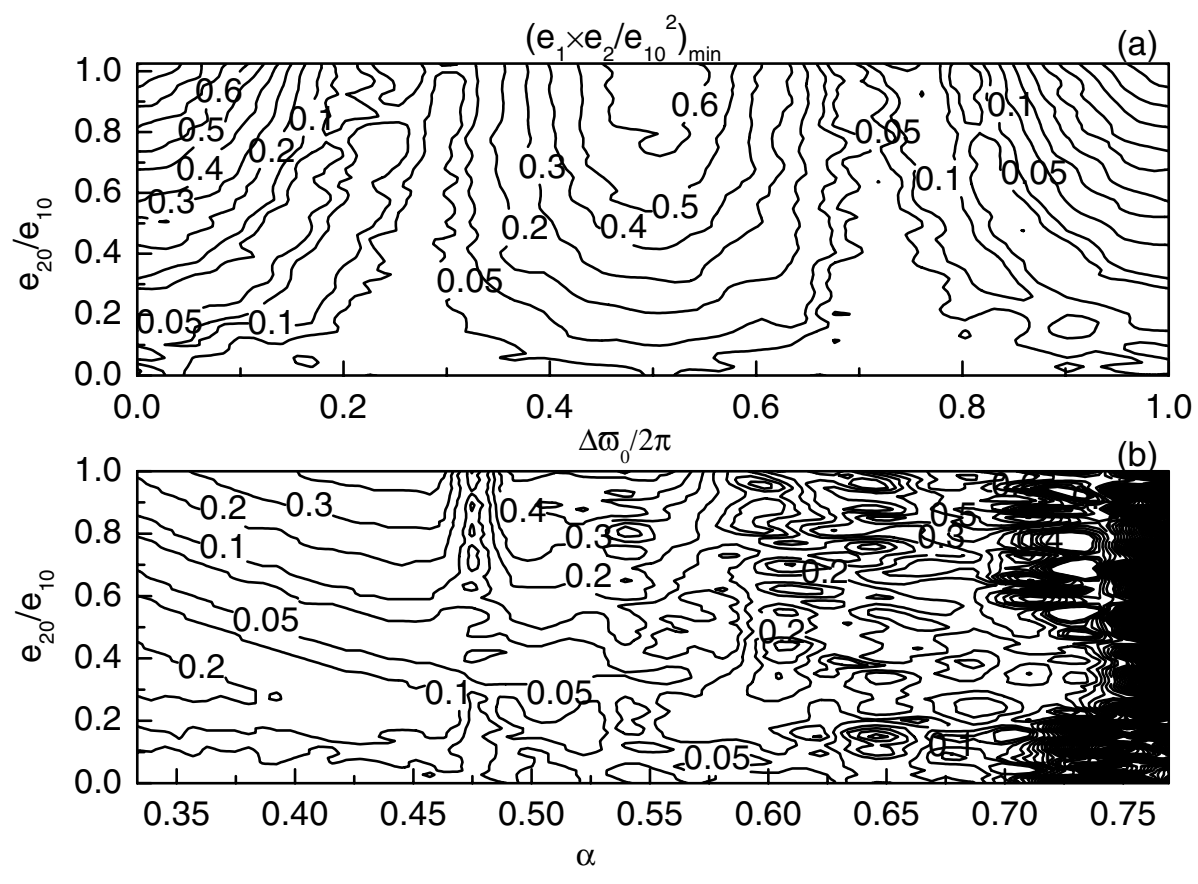

Figure 3. Results of general three-body simulations. (a) Contours of $\eta$ changed with $e_{20} / e_{10}$ and $\Delta \varpi_{0}(\alpha=0.5)$. (b) Contours of $\eta$ changed with $e_{20} / e_{10}$ and $\alpha\left(\Delta \varpi_{0}=2 \pi / 9\right)$.

runaway gas-accretion could be very fast, with a mass-doubling time of a Jupiter-massplanet being few thousand years (Zhou \& Lin 2007). Under the fast grow of planet-mass increasing, the previously stable system becomes unstable if the previous distance is small compared with the enlarged Hill radius of growing planet. Thus the newly formed gas giant can perturb the previous formed gas giant, and their mutual interaction may lead to the ejection of one of the gas giants.

Consider a model planetary system similar to upsilon And (Butler et al. 2006) with a $1.3 M_{\odot}$ star and two planets 1,2 . The mass of planet 1 and 2 is 1.94 and 3.95 Jupiter mass and semi-major axis is 0.83 and $3 \mathrm{AU}$, respectively. In this model planet 2 has consumed the gas in its feeding zone ( 1 Hill radius $=0.33 \mathrm{AU})$. Thus when we fix the additional planet 3 at $4.05 \mathrm{AU}$ ( 1 Hill radius $=0.28 \mathrm{AU}$ ), there is still enough gas for the accretion process which leads planet 3 grow from an initial mass of 10 Earth-mass to a Jupiter-mass. In the simulations we let its mass accrete according to a formula of Ikoma et al (2002) in a timescale of $10^{5}$ years. For simplicity, we let all the three planets on circular and coplanar orbits initially.

We perform 120 sets of numerical integrations by varying initial longitude of planets in the system. Figure 5 shows a typical evolution. From figure 5 we know that in the beginning the evolution of the system is regular. But after planet 3 reaching a Jupiter mass, it is ejected and planet 2 get a large eccentricity while the eccentricity of planet 1 is oscillating near zero, resulting a system undergo near-separatrix motion. 

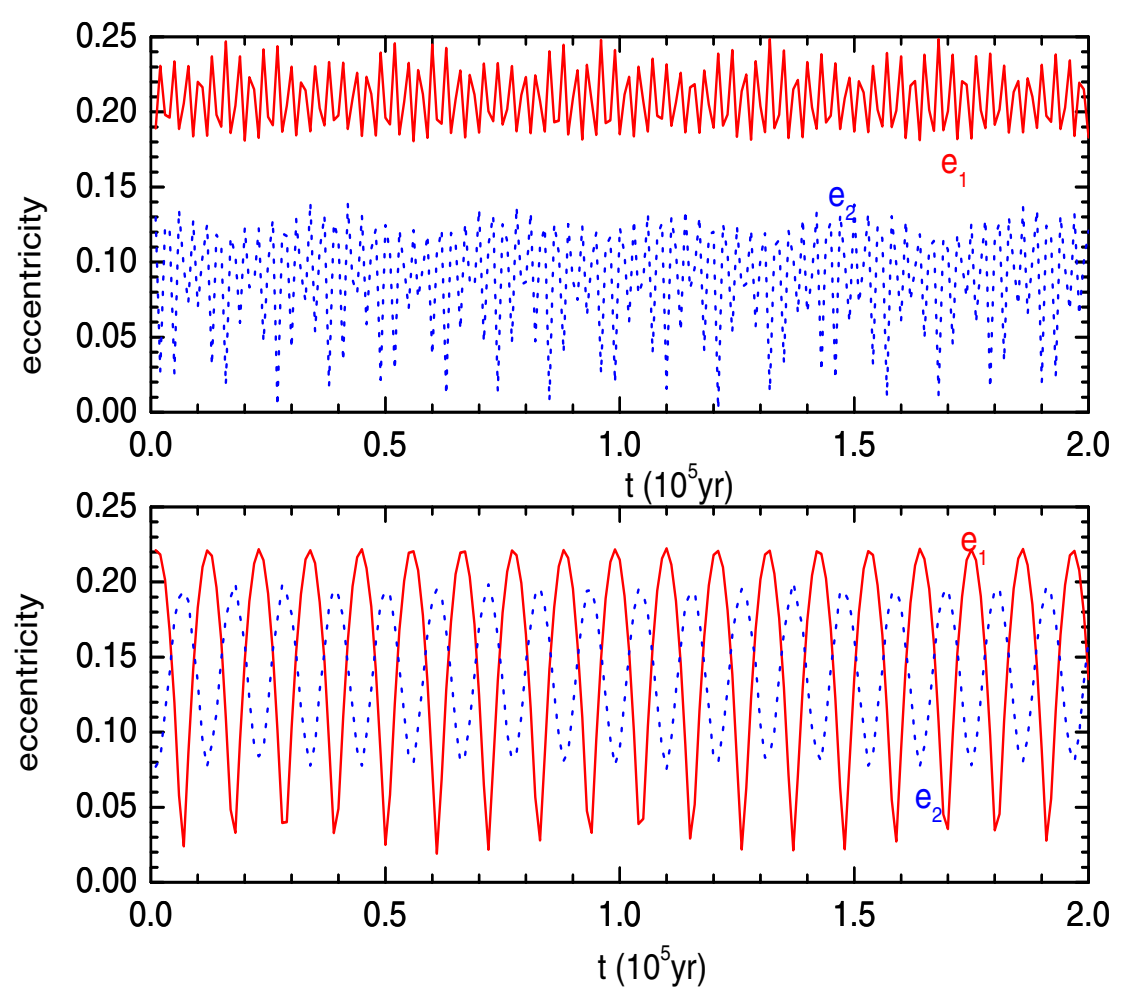

Figure 4. (a) Eccentricity evolution of the system undergo mean motion resonance. (b) Eccentricity evolution of the system $a_{20}=2.3 \mathrm{AU}$

For the 120 sets of simulations, 7 cases show strong chaotic motion with $e \sim 0.6$, so that the system will tend to be destroyed after a very long time, leaving one planet finally. For the left 113 cases, 57 cases results in all the three planets survived after the third planet accreting to Jupiter mass, and 56 cases with one planet scattered and two planets left. So there are $56+57 \times 2=170$ planet pairs for our 113 sets of integration. Our statistics shows, among the 170 planet pairs, 145 ( 85\%) pairs undergo near-separatrix motion (the eccentricity of at least one planet in the pair oscillate near zero), 21 cases are in circular motion, only 4 systems are in libration motions ( 2 systems with pericenters of the two planets aligned, and 2 anti-aligned). The ratio of near-separatrix motion is large enough as compared with the observed system (over 40\%, Barnes 2008). Most of the near-separatrix motion occur in the cases that the third planet has not been scattered out, and it's perturbation to the inner two planets is too small, thus their eccentricities have changed slightly (with maximum values $<0.1$ ).

\section{Conclusions and discussions}

Statistics about recent observed multiple-planet systems indicate that near-separatrix motion could be quite common. Based on the linear secular dynamics, a two-planet system will undergo near-separatrix motion provided one of the planet in near circular orbit initially. Due to either dynamical friction or tidal damping of the gas disk, a protoplanet will most possibly be on near circular orbits after they formed. However, two procedures may disrupt the near circular configurations: (1) migration of protoplanet in a gaseous 


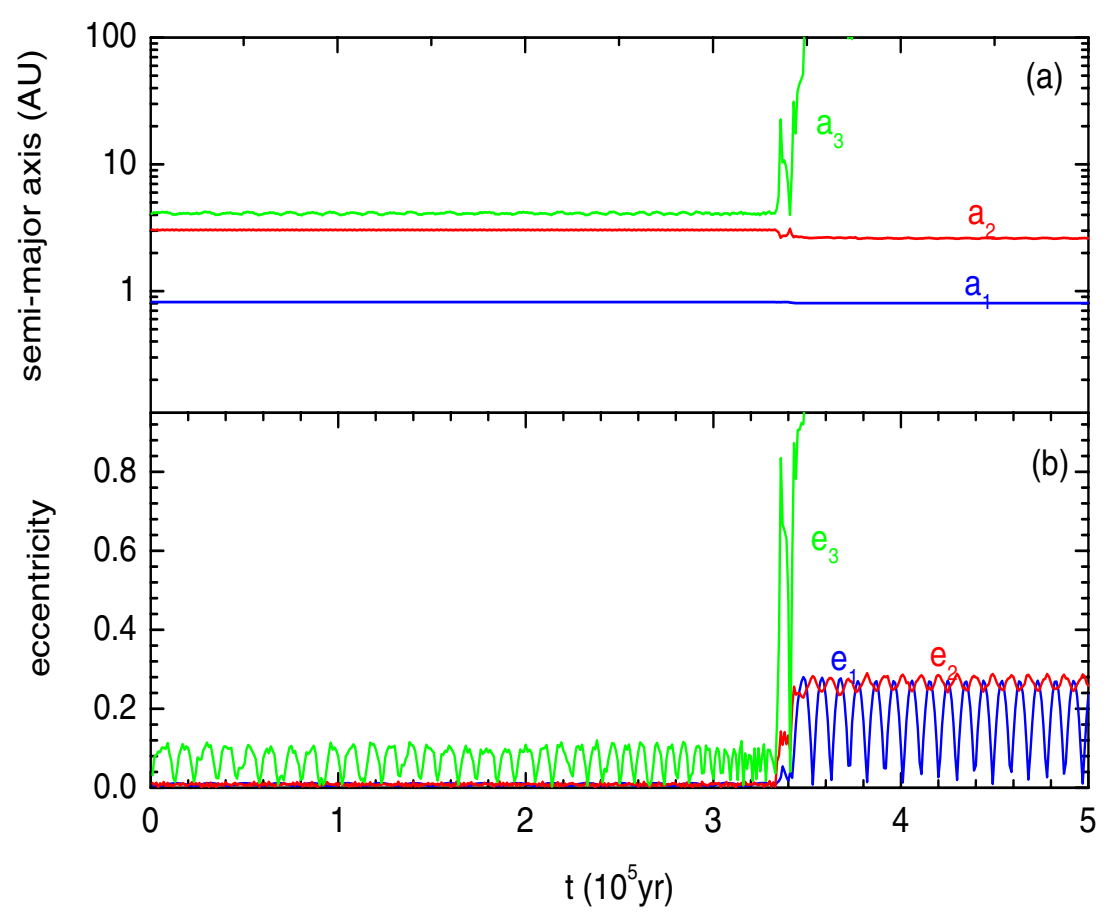

Figure 5. (a) Semi-major axis evolution of the 4-body system. (b) Eccentricity evolution of the 4-body system, $e_{1}$ undergoes large amplitude oscillation, will $e_{2}$ undergoes small amplitude oscillation.

disk; (2) scattering among planets. As we know, type II migration which leads to the resonance trapping of two gas giants may cause the increase of eccentricity of both orbits (e.g., Kley 2004). Unless in some rare cases, if type-II migration ceases just at the epoch when the two gas giants being captured into resonances, the system will be in a nearseparatrix motion (Sándor \& Kley 2007). For the scattering model, although the results of Barnes (2008) indicate that the final results can not produce the observed high rate of near-separatrix motion, they neglected those unsuccessfully scattered affairs, which must occurr in the real cases. The window that the third planet can be scattered is small, while most of cases the third planet is not ejected out, resulting in a slight changing of eccentricities of three plants and the near-separatrix motion remains. Our preliminary result in this report indicates this could be a possible explanation of the high probability of near-separatrix motion of multiple planets system. More detailed investigation on this proposition will be done in the future.

We thank Dr. R. Barnes and W. Kley for very helpful discussions. This work is supported by NSFC(10778603), National Basic Research Program of China(2007CB4800).

\section{References}

Barnes, R. \& Greenberg, R. 2006, ApJ, 638, 478

Barnes, R. \& Greeberg, R. 2006, ApJ, 652, L53

Barnes, R. 2008 , this issue.

Buter, R. P., et al. 2006, ApJ, 646,505

Ford, E. B., Lystad, V., \& Rasio, F. A. 2005, Nature, 434, 873 
Gladman, B. 1993, Icarus, 106, 247

Ikoma, M., Nakazawa, K., \& Emori, H. 2000, ApJ, 537, 1013

Kley, W., Peita, J., \& Bryden, G. 2004, A \& A, 414, 735

Laughlin, G., Chambers, J., \& Fischer, D. 2000, ApJ, 579, 455

Marcy, G., et al. 2005, PThPS, 158, 24

Papaloizou, J. C. B. \& Terquem, C. 2006, RPPh, 69, 119

Pollack J. B. et al. 1996, Icarus, 124, 62

Sándor, Zs., Kley, W. 2007, A \& A, 472, 781

Zhou, J. L. \& Sun, Y. S. 2003, ApJ, 598, 1290

Zhou, J. L. \& Lin, D. N. C 2007, ApJ, 666, 447

Zhou, J. L., Lin, D. N. C. \& Sun, Y. S. 2007, ApJ, 666, 423 